\title{
Praktische Erfahrungen mit Online-Registern
}

\author{
Simon Fleischlia, Marc Otto ${ }^{b}$, Max Giger ${ }^{c}$ \\ ${ }^{a}$ M Sc UZH, IT Architekt, pnn pharma nation network AG; ${ }^{b}$ Dr. oec. publ., Verwaltungsrat pnn pharma nation network $A G$; ${ }^{c}$ Dr. med.
}

Die systematische Erfassung von Gesundheitsdaten bzw. Gesundheitsregistern ist Voraussetzung für die Effizienzsteigerung und Qualitätssicherung des Gesundheitswesens. Die Daten zu Krankheiten und Therapien sollten umfassend und gezielt auf die Fragestellungen gesammelt werden. Der Persönlichkeitsschutz der registrierten Personen muss gewahrt werden. Im Jahr 2012 wurden in der Schweiz rund 100 Register erfasst [1]. Der zeitliche Aufwand zur Erfassung der Daten hängt von deren Umfang ab. Ärzte, Pflegende und Therapierende lehnen die Teilnahme an Registern oft $a b$, da sie vor einem weiteren zeitlichen administrativen Aufwand zurückschrecken oder den Datenschutz gefährdet sehen. Anhand zweier Online-Register werden die Voraussetzungen für den Aufbau, der zeitliche Aufwand für die Datenerfassung und die Möglichkeit aufgezeigt, die Vorschriften des Datenschutzes einzuhalten.

\section{Die beiden Online-Register}

Das erste Register wurde aufgrund einer Einschränkung der Kostenerstattung durch die Obligatorische Krankenversicherung (Limitatio in der Spezialitätenliste) eines Arzneimittels aufgebaut. Es sollte zeigen, wie viele $\mathrm{Pa}-$ tientinnen und Patienten mit wie vielen Infusionen dieser pharmazeutischen Spezialität und in welcher Dosierung behandelt wurden. Zudem wurde dokumentiert, bei wie vielen Patienten die Behandlung wiederholt wurde. Beim zweiten Register ging es um die prospektive Erfassung (Zeitraum und Maximalzahl der Patienten begrenzt) von Patientinnen und Patienten mit eingeschränkter Nierenfunktion, die wegen Diabetes II unter oralen Antidiabetika standen.

In beiden Registern wurden die Daten in einer OnlineDatenbank gesammelt. Beim ersten Register erfassten 33 Datenlieferanten während 24 Monaten, beim zweiten Register 109 Datenlieferanten während sieben Monaten Patienten. Die 33 Datenlieferanten des ersten Registers waren Ärzte aus 4 Universitäts-, 9 Kantons-, 5 Regionalund 2 Privatspitälern sowie 15 Einzel- und Gruppenpraxen. Die 109 Datenlieferanten des zweiten Registers waren Ärztinnen und Ärzte aus dem ambulanten Versorgungssektor.

Um die persönlichen Daten der Patienten zu schützen, hat man sich für eine Verschlüsselung der Patienten-
Expériences pratiques avec les registres en ligne A l'exemple de deux registres en ligne, il a été possible de démontrer qu'il fallait peu de temps pour saisir des données tout en respectant les exigences de protection des données par pseudonymisation. Dans les deux registres en ligne, les médecins ont saisi les données de patients sous traitement médicamenteux. Le premier registre a permis de déterminer le nombre total de patients traités au moyen de produits thérapeutiques hautement spécialisés. Pour chaque patient, 16 paramètres ont été saisis. II a fallu en moyenne 15 minutes au médecin pour enregistrer les données. Le second registre a documenté le nombre de patients souffrant d'un diabète de type II avec insuffisance rénale et traités par antidiabétiques oraux. 44 paramètres ont été saisis. Pour enregistrer la totalité des données, les médecins ont eu besoin de $7 \mathrm{mi}$ nutes en moyenne. La grande différence dans le temps de saisie moyen par patient est plutôt à mettre sur le compte du nombre différent de patients par session. Il est en effet plus efficace d'enregistrer simultanément les données de plusieurs patients.

daten entschieden. Dafür teilte das System jedem Patientenfall ein Pseudonym zu. Die Nachfrage bei kantonalen Ethikkommissionen ergab, dass die Datenerhebung in beiden Registern keiner Beurteilung bzw. Bewilligung durch eine kantonale Ethikkommission bedurfte.

Die Datenlieferanten beider Register rekrutierten die Auftraggeberinnen (Pharmafirmen). Beim ersten Register rekrutierten die Mitarbeitenden des Aussendienstes Ärzte als Datenlieferanten und schlossen entsprechende Verträge ab. Die Vertragsabschlüsse wurden dann der Zentrale des Registers gemeldet, welche die persönlichen Accounts erfasste und die teilnehmenden Ärzte via E-Mail und Post (Passwort, Kurzinformation) zur Erfassung einlud.

Beim zweiten Register rüstete die Datenzentrale die Mitarbeitenden des Aussendienstes mit einer Anzahl eineindeutiger Aktivierungscodes aus, die bei Vertragsabschluss den Ärzten übergeben wurden. Die Ärzte registrierten sich danach online, mussten diesen Aktivierungscode eingeben und waren dadurch als Datenlieferanten identifiziert. Bei beiden Registern wurde mit jedem Datenlieferanten ein Vertrag geschlossen. Die Aufwandsentschädigung inklusive Aktenstudium war als Pauschale pro Patientenfall festgelegt. 


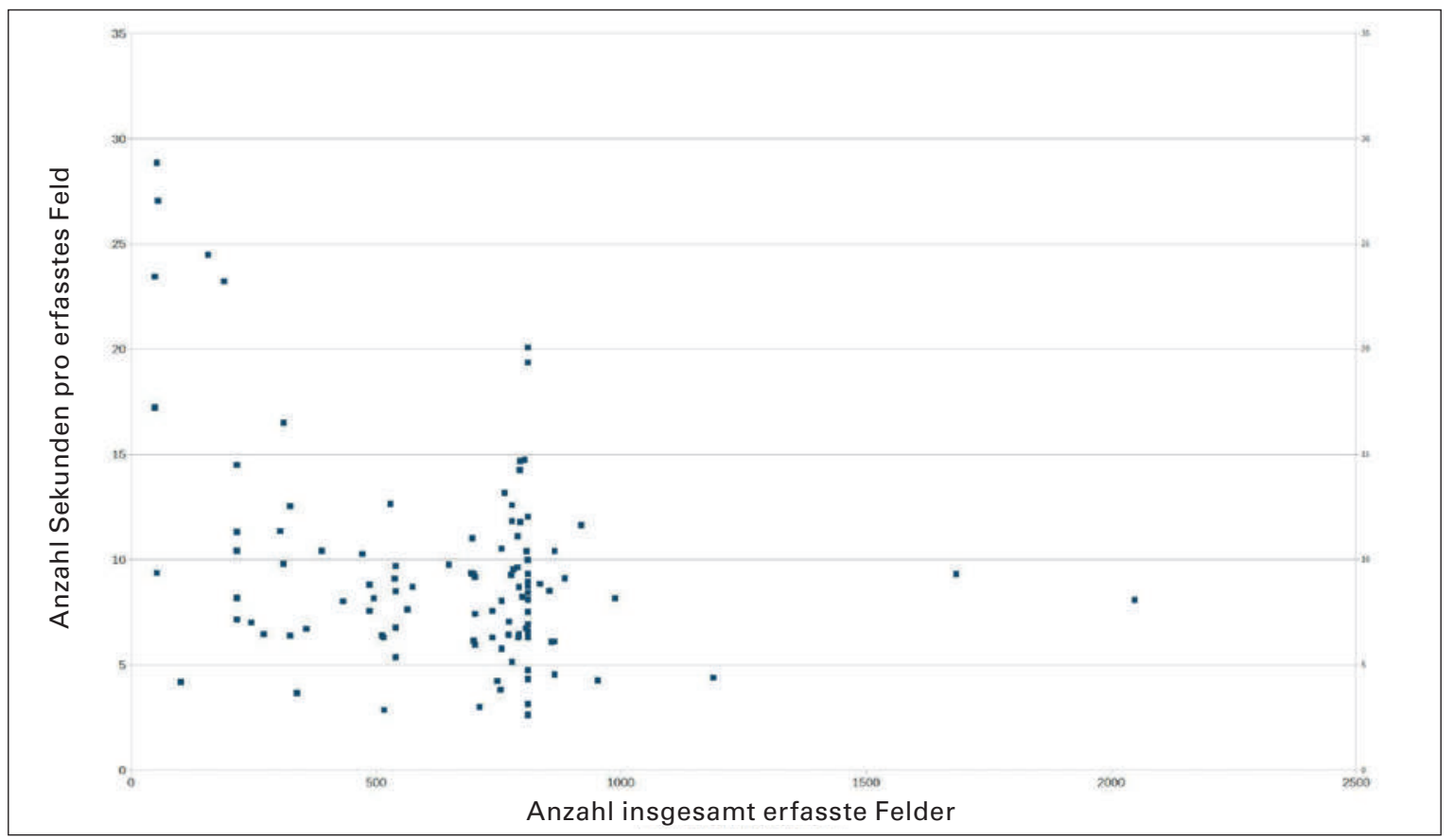

Abbildung 1: Zeitaufwand und Anzahl erfasster Felder pro Datenlieferant.

\section{Ansatz und Erfahrungen zu Datenqualität}

Mit fünf Massnahmen wurde versucht, eine maximale Abdeckung zu erreichen: niederschwellige Ersterfassung jedes Patientenfalls, während der üblichen Büroarbeitszeiten erreichbarer telefonischer Benutzersupport, selbsterklärende Benutzerführung, Einsatz von Auswahlfeldern und vorsichtiger Einsatz rigider automatischer Sofortvalidierungen.

Fehlten einzelne Daten oder waren einzelne Eingaben nicht plausibel, wurde per E-Mail beim Datenlieferanten nachgefragt. Für diesen waren in den Erfassungsmasken die entsprechenden Datenfelder farblich markiert. Nun musste er entweder die Eingabe korrigieren oder bestätigen, dass kein Eingabefehler bestand. Selbstverständlich blieb jede Veränderung im System dokumentiert.

Kriterium für die automatischen Konsistenztests war beim ersten Register der Vergleich der erfassten Dosis mit dem aufgrund des Körpergewichts errechneten Dosiswert. Beim zweiten Register waren bei Feldern mit numerischen Eingaben die Konsistenztests mit grossen Wertebereichen gleich im Erfassungstool integriert, um offensichtliche Fehleingaben sofort abzufangen.

Im Rahmen periodischer Bestandesanalysen wurden bei eröffneten Patientenfällen, zu denen innerhalb einer bestimmten Zeit keine neuen Daten eingingen, dem betreffenden Datenlieferanten ein automatisch generiertes, personalisiertes Erinnerungsschreiben («Reminder») zugestellt mit der Bitte, die Daten dieser Fälle zu komplettieren.
Als effektiver erwies sich periodisches Nachfragen per Telefon. Dies kam vor allem bei Datenlieferanten mit grösseren Datenlücken oder Datenlieferanten mit nahezu vollständig erfassten Datensätzen sowie bei Datenlieferanten, die nicht auf die «Reminders» reagiert hatten, zum Einsatz. Zusätzlich wurde die Datensammlung von den Aussendienstmitarbeitern der Auftraggeberinnen unterstützt. Sie klärten vor Ort Unsicherheiten und leiteten offene Fragen der Datenlieferanten an die Zentrale weiter.

Da es für die «Reminders» entscheidend war, dass die Patientenfälle überhaupt im System erfasst sind, war eine niederschwellige Ersterfassung jedes Patientenfalls zentral, d.h., zur Ersterfassung eines Patienten waren lediglich zwei Felder Pflichteingaben. Diese Pflichteingaben waren Initialen und Geburtsjahr des Patienten, also die Angaben zur eineindeutigen Identifikation des verschlüsselten Falls. Alle anderen Datenfelder konnten leergelassen werden. Da Initialen und Geburtsjahr der eineindeutigen Identifikation dienten, konnten diese im Nachhinein nicht mehr durch den Datenlieferanten verändert werden.

Die Benutzerführung wurde möglichst einfach und selbsterklärend gestaltet. So reichten im Wesentlichen zwei Formularseiten, um die Daten eines Patientenfalls zu erfassen. Die Patientenliste zeigte auf einen Blick alle erfassten Fälle, unterschiedliche Hintergrundfarben zeigten bei jedem einzelnen Fall, wie vollständig die Daten bereits erfasst waren.

Sobald ein Patientenfall im System auch nur teilweise erfasst worden war, konnte er von der Zentrale über die 
gesamte Zeit bis zur vollständigen Eingabe aller Pflichtparameter aktiv begleitet werden.

\section{Datenerfassung und Ergebnisse}

Im ersten Register mussten 9 Parameter zum Patienten selbst und 7 Parameter zu einer einzelnen Behandlung erfasst werden. Kam es zu einer erneuten Behandlung, wurde diese mit weiteren 7 Parametern dokumentiert. Es wurden 199 Patienten erfasst, wovon für 194 vollständig dokumentierte Patienten insgesamt 199 Behandlungen erfasst wurden; bei 5 Patienten wurde die Behandlung also wiederholt. 3 Behandlungen von 3 Patienten wurden nur unvollständig erfasst.

\section{Die vollständige Eingabe der Daten dauerte im Durchschnitt sieben Minuten.}

Im zweiten Register wurden pro Patientenfall 52 Parameter abgefragt, wovon 44 Pflichtangaben waren, d.h., nur falls diese Pflichtangaben komplett ausgefüllt waren, galt der Patientendatensatz als vollständig. Bei den Datensätzen für Anamnese (24 Parameter) und Medikation (11 Parameter) war nur je ein Feld kein Pflichtfeld. Bei den Laborwerten galten einzig $\mathrm{HbA}_{1 \mathrm{c}}$ und SerumKreatinin als Pflichtangabe. Angaben von Albumin/ Kreatinin-Quotient im Urin, Mikroalbuminurie, Gesamtcholesterin, HDL, LDL, Triglyzeride waren freiwillig. Damit wollte man vermeiden, dass die erfassenden Ärzte keine Zeit mit Eingaben wie «nicht erhoben» verlieren. Es wurden 1359 Patientenfälle vollständig erfasst. 19 Fälle wurden nur unvollständig erfasst.

1 Strub S, Stettler P, Künzi K. Forum medizinische Register Schweiz: Bericht «Medizinische Registerlandschaft Schweiz». Bern: Büro Bass; 2012.

2 www.siris-implant. ch/de/Fachinforma tion/Datenfluss (2.5.2015).

3 Hoffman A, Montgomery R, Aubry W, Tunis SR. How Best To Engage Patients, Doctors, And Other

Stakeholders In Designing Comparative Effectiveness Studies. Health Aff. 2010;29:1834-41.

Korrespondenz: Dr. oec. publ. Marc Otto pnn pharma nation network AG

Kirchgasse 42 CH-8001 Zürich
Bei beiden Registern blieben knapp 1,5 Prozent aller dokumentierten Fälle bzw. Therapien trotz Nachfrage «unvollständig».

Im ersten Register benötigten die 33 Datenlieferanten im Durchschnitt 14 Minuten und 50 Sekunden (Median 14 Minuten 28 Sekunden), um einen Patientenfall vollständig zu erfassen. Diese Zeit wurde errechnet, indem die in den Logfiles der Online-Plattform festgehaltene gesamte Online-Aufenthaltsdauer durch die Anzahl vollständig erfasster Patienten geteilt wurde. Im ersten Register hatten sich die Datenlieferanten pro vollständig erfassten Patientenfall im Durchschnitt 1,88 Mal ins System eingeloggt (Median 1,70).

Im zweiten Register hatten sich die Datenlieferanten im Durchschnitt 0,48 Mal ins System eingeloggt (Median $0,46)$, um einen Patientenfall vollständig zu erfassen; es wurden also in einer Erfassungssession mehr als zwei Patientenfälle vollständig erfasst.

Aus den Logfiles wurde aufgrund des Online-Aufenthaltes und der erfassten Felder berechnet, wie viele Datenfelder jeder Datenlieferant tatsächlich ausgefüllt hatte und wie viel Zeit er durchschnittlich fürs Ausfüllen aller Felder aufgewendet hatte (Abb. 1). Jeder Punkt entspricht einem Datenlieferanten.
Im zweiten Register benötigten die Datenlieferanten im Durchschnitt 7 Minuten und 18 Sekunden (Median 7 Minuten 14 Sekunden), um einen Patientenfall vollständig zu erfassen. Im Durchschnitt benötigten die Datenlieferanten hier 8,4 $\pm 4,8$ Sekunden, um ein Feld auszufüllen.

\section{Diskussion}

Bei der Rekrutierung von Datenlieferanten helfen eine gute Planung und ein einfacher Prozess für deren Registrierung. Der Mechanismus der Aktivierungscodes hat sich im zweiten Register sehr bewährt und diese Phase im Vergleich zum ersten deutlich vereinfacht.

Wichtig sind zudem eine gute und selbsterklärende Benutzerführung sowie eine zeitnahe, systematisierte Validierung der erfassten Daten und ein gut erreichbarer Benutzersupport.

Die gesamte Zeit zur Erfassung eines Patientenfalls war vor allem abhängig von der Anzahl erfasster Parameter. Pro Parameter bzw. ausgefülltes Feld wandten die Datenlieferanten des zweiten Registers im Durchschnitt 8,4 Sekunden auf. Können pro Erfassungssitzung mehrere Patientenfälle erfasst werden, wird die Arbeit effizienter und die gesamte Erfassungszeit kürzer. Müssen Daten zu verschiedenen Zeiten - u.a. Aufnahme einer Behandlung, Abschluss einer Behandlung - eingegeben werden, verlängert dies die gesamte Erfassungszeit. Zudem vermuten wir, dass die Erfassungszeit beim zweiten Register kürzer war, da die Datenlieferanten dank der Konsistenztests von Zahlenfeldern gewisse Fehler bei der ersten Eingabe bereits korrigierten.

Die total aufgewendete Zeit betrug 891 Sekunden für die Erfassung eines Therapiezyklus im ersten und 434 Sekunden für die Erfassung der Daten im zweiten Register. $\mathrm{Zu}$ diesen elektronisch erfassten Zeiten müssen noch 60-80 Sekunden pro Fall fürs Öffnen und Schliessen des Falls addiert werden. Der totale, effektiv erfasste Zeitaufwand pro Patientenfall des zweiten Registers beträgt wie derjenige der elektronischen Erfassung der klinischen Daten bei Implantation einer Hüft- oder Kniegelenksprothese im obligatorischen Schweizerischen Implantat-Register (Siris) [2] weniger als zehn Minuten.

Gelingt es, einen guten Datensatz zu definieren, so sind Online-Register ein effizientes und für die Datenlieferanten mit wenig Zeitaufwand verbundenes Mittel, um ein aussagekräftiges Register zu etablieren, zu betreiben und auszuwerten. Online-Register - wie die dargestellten - eignen sich gut zur prospektiven Erfassung diagnostischer und therapeutischer Interventionen unter sog. Alltagsbedingungen bzw. zur Datenregistrierung im Bereich der "Comparative Effectiveness Research" [3]. So könnten mit relativ geringem Aufwand Register geführt werden zu Off-Label-Use oder zu Einsatz, Verträglichkeit und Nutzen neuer Arzneimittel, die einzig aufgrund von Phase-II-Studien zugelassen wurden. 